\title{
Ononis spinosa alleviated capsaicin-induced mechanical allodynia in a rat model through transient receptor potential vanilloid 1 modulation
}

\author{
Sahar Majdi Jaffal ${ }^{1}$, Belal Omar Al-Najjar ${ }^{2,3}$, and Manal Ahmad Abbas ${ }^{3,4}$ \\ 'Department of Biological Sciences, Faculty of Science, The University of Jordan, Amman, Jordan \\ 2Department of Pharmaceutical Sciences, Faculty of Pharmacy, Al-Ahliyya Amman University, Amman, Jordan \\ ${ }^{3}$ Pharmacological and Diagnostic Research Center, Al-Ahliyya Amman University, Amman, Jordan \\ ${ }^{4}$ Department of Medical Laboratory Sciences, Faculty of Allied Medical Sciences, Al-Ahliyya Amman University, Amman, Jordan
}

Received January 2, 2021

Revised April 7, 2021

Accepted April 8, 2021

Handling Editor: Sang Hun Kim

\section{Correspondence}

Sahar Majdi Jaffal

Department of Biological Sciences,

Faculty of Science, The University of

Jordan, Amman 11942, Jordan

Tel: +962787924254

Fax: +96265300253

E-mail: sjaff333@gmail.com
Background: Transient receptor potential vanilloid 1 (TRPV1) is a non-selective cation channel implicated in pain sensation in response to heat, protons, and capsaicin (CAPS). It is well established that TRPV1 is involved in mechanical allodynia. This study investigates the effect of Ononis spinosa (Fabaceae) in CAPS-induced mechanical allodynia and its mechanism of action.

Methods: Mechanical allodynia was induced by the intraplantar (ipl) injection of 40 $\mu g$ CAPS into the left hind paw of male Wistar rats. Animals received an ipl injection of $100 \mu \mathrm{g} 0$. spinosa methanolic leaf extract or $2.5 \%$ diclofenac sodium 20 minutes before CAPS injection. Paw withdrawal threshold (PWT) was measured using von Frey filament 30, 90, and 150 minutes after CAPS injection. A molecular docking tool, AutoDock 4.2, was used to study the binding energies and intermolecular interactions between 0 . spinosa constituents and TRPV1 receptor.

Results: The ipsilateral ipl injection of $O$. spinosa before CAPS injection increased PWT in rats at all time points. 0 . spinosa decreased mechanical allodynia by 5.35-fold compared to a 3.59-fold decrease produced by diclofenac sodium. The ipsilateral pretreatment with TRPV1 antagonist (300 $\mathrm{gg}$ 4-[3-Chloro-2-pyridinyl]$N$-[4-[1,1-dimethylethyl] phenyl]-1-piperazinecarboxamide [BCTC]) as well as the $\beta 2$-adrenoreceptor antagonist (150 $\mu \mathrm{g}$ butoxamine) attenuated the action of $O$. spinosa. Depending on molecular docking results, the activity of the extract could be attributed to the bindings of campesterol, stigmasterol, and ononin compounds to TRPV1.

Conclusions: 0 . spinosa alleviated CAPS-induced mechanical allodynia through 2 mechanisms: the direct modulation of TRPV1 and the involvement of $\beta 2$ adrenoreceptor signaling.

Key Words: Butoxamine; Capsaicin; Fabaceae; Hyperalgesia; Molecular Docking Simulation; Neuralgia; Ononis; Pain; Stigmasterol; TRPV Cation Channels. (c) This is an open-access article distributed under the terms of the Creative Commons Attribution Non-Commercial License (http://creativecommons.org/licenses/by-nc/4.0/), which permits unrestricted non-commercial use, distribution, and reproduction in any medium, provided the original work is properly cited.

(c) The Korean Pain Society, 2021
Author contributions: Sahar Majdi Jaffal: Investigation, Writing/manuscript preparation, Analysis for results; Belal Omar Al-Najjar: Investigation; Manal Ahmad Abbas: Investigation. 


\section{INTRODUCTION}

Transient receptor potential vanilloid 1 (TRPV1) is a nonselective cation channel involved in inducing the symptoms of pain in response to heat, protons, and capsaicin (CAPS) [1]. It is expressed in the dorsal root ganglia (DRGs), the cell bodies of the nociceptors extending to $\mathrm{A} \delta$ - and Cfibers [2]. It is well established that the activation of this channel is responsible for mechanical allodynia [3] and thermal hyperalgesia [4]. Several inflammatory mediators modulate TRPV1 indirectly through binding to their receptors and formation of second messengers that activate TRPV1, leading to its phosphorylation $[5,6]$. This stimulation increases the probability of activating the open state of this channel or its translocation to the plasma membrane $[5,6]$.

Despite the fact that there are several analgesics in the market, many of them have side effects [7]. Accordingly, there is an increasing need for the development of new and safe drugs. Ononis spinosa is a spiny herb with trifoliate serrate leaflets, non-sticky hanging stems, and pink to violet flowers [8]. It is common in Asia, Europe, and Africa [9]. This plant belongs to Fabaceae family and is used for the treatment of inflammatory diseases, kidney stones [10], wound healing [11], and toothache [12]. Also, the antinociceptive effects of $O$. spinosa were reported previously using thermal and chemical models of pain $[10,13]$.

Mechanical allodynia is defined as a painful sensation that results from innocuous stimuli such as touch [14]. It can be produced by the intraplantar (ipl) injection of CAPS (the pungent ingredient in chili pepper and the agonist of TRPV1 channel) into the hind paw of animal $[2,3]$. The nociceptive flexion reflex is quantified by applying an increasing mechanical force into the rat's hind paw [15]. Since previous studies have not examined the effect of $O$. spinosa in any model of mechanical allodynia, the present study was designed to evaluate this effect in rats. In addition, the mechanism of action of $O$. spinosa was investigated using a TRPVl antagonist and an antagonist for $\beta 2$ adrenoreceptor. Molecular modelling was, also, used to predict the binding affinities of the active constituents in $O$. spinosa extract to TRPV1 receptor.

\section{MATERIALS AND METHODS}

\section{Drugs}

Butoxamine hydrochloride, a selective $\beta 2$ adrenoreceptor antagonist, was purchased from Sigma-Aldrich, St. Louis. Diclofenac sodium, a non-steroidal anti-inflammatory drug (NSAID), was brought from Novartis, El-
Salvador. CAPS, a TRPV1 agonist, and BCTC [4-[3-Chloro-2-pyridinyl]- $N$-[4-[1,1-dimethylethyl] phenyl]1-piperazinecarboxamide], a TRPV1 antagonist, were brought from Tocris Bioscience, Bristol, United Kingdom. Methanol and ethanol were purchased from Scharlau, Spain. CAPS was dissolved in virgin olive oil, Al-Jazzazi Mill, Salt, Jordan, while BCTC was dissolved in absolute ethanol. Diclofenac sodium (2.5\%), butoxamine, and $O$. spinosa extract were dissolved in sterile normal saline. All drugs were freshly prepared and injected (ipl) in $100 \mu \mathrm{L}$ total volume.

\section{Plant collection and extraction}

O. spinosa was collected from Subaihi, Al-Balqa, Jordan in July 2017. The collected plant was identified by Prof. Barakat Abu-Irmaileh, Faculty of Agriculture at the University of Jordan. A voucher specimen was kept at the Graduate Studies Laboratory in Al-Ahliyya Amman University. Methanolic extract of $O$. spinosa leaves was obtained by maceration of groud leaves in $96 \%$ methanol. After filtration, the methanol was evaporated using a rotary evaporator at a temperature not exceeding $45^{\circ} \mathrm{C}$, then was stored at $-20^{\circ} \mathrm{C}$. The extract was freshly prepared before use and was dissolved in sterile normal saline.

\section{Experimental animals}

All procedures were performed according to the Guide for the Care and Use of Laboratory Animals published by the National Institutes of Health and comply with the guidelines of the International Association for the Study of Pain. Ethical approval (No. AAU-2/4/2018) for conducting the work was obtained from Al-Ahliyya Amman University. Male Wistar rats (250-300 g) were brought from the animal house at Al-Ahliyya Amman University, Jordan, and were kept at $23 \pm 2^{\circ} \mathrm{C}$ with an alternating 12 hour light-dark cycle. Food and water were provided ad libitum. The animals were allowed to adapt to the laboratory conditions for at least 2 hours before test.

\section{Treatment of animals}

The animals were divided into 8 groups (with 8 rats in each group). Mechanical allodynia was induced by the ipl injection of $40 \mu \mathrm{g}$ CAPS into the left hind paw of the animals. Group I received ipl injection of vehicle only (control group). Group II received ipl injection of CAPS. Group III received ipl injection of $100 \mu \mathrm{g}$ plant extract into the left hind paw 20 minutes before the ipl injection of CAPS. Group IV, the positive control group, received 2.5\% diclofenac sodium 20 minutes prior to CAPS injection. The dose 
and time of injecting diclofenac sodium was chosen pursuant to the study of Nozadze et al. [16]. Groups V and VI received $300 \mu \mathrm{g}$ BCTC and $150 \mu \mathrm{g}$ butoxamine 30 minutes prior to the injection of plant extract, respectively followed by CAPS injection. Groups VII and VIII received the antagonists BCTC and butoxamine 30 minutes before CAPS injection, respectively. All drugs, vehicle, and plant extract were injected in a volume of $100 \mu \mathrm{L} /$ paw into the left paw of the rats.

\section{Measurement of CAPS-induced mechanical allodynia}

The experimental rats were allowed to acclimatize for 1 hour in wire mesh-floor cages covered with perforated lids. Baseline withdrawal thresholds were assessed in the first day to ensure that the animals have equivalent baseline readings before starting the test. Paw withdrawal threshold (PWT) of the ipsilateral (left hind paw) and the contralateral (right hind paw) hind paws were measured using handmade von Frey filament (pursuant to Ferrier et al. [17]) 30, 90, and 150 minutes after CAPS injection. Briefly, a thin filament was used to press against the plantar surface of the animal's hind paw. The filament was pressed once against the hind paw of the animal at each measured time point. A sharply withdrawn paw was regarded as a positive response. A cut-off mass unit of $80 \mathrm{~g}$ was used in this experiment according to Ferrier et al. [17]. Also, the behavior of animals was observed and recorded.

\section{Molecular docking}

The X-ray crystallographic structure of TRPV1 (PDB ID: 5IS0) was selected from Protein Data Bank (https://www. rcsb.org/) [18]. The crystal structure is co-crystallised with the TRPV1 antagonist, capsazepine [19]. The molecular docking tool, AutoDock 4.2 (The Scripps Research Institute, La Jolla, CA) was used to study the intermolecular interactions and binding energies of the proposed compounds found in $O$. spinosa leaf extract.

\section{Ligand preparation}

The two dimensional (2D) chemical structures of the major compounds found in $O$. spinosa leaf extract were generated by ACD/ChemSketch software [20]. All compounds were saved as mol files by ChemSketch software then converted to pdb files after minimization utilizing BIOVIA DS Visualizer [21]. The pdb files were uploaded in AutoDockTools and the gasteiger charges were added to each compound. These files were converted to AutoDock pdbq format, indicating all possible rotatable bonds in the structure.

\section{Protein preparation}

The X-ray crystallographic structure of TRPV1 (PDB ID: 5IS0) was downloaded from the Protein Data Bank. According to the co-crystallised ligand (capsazepine), the intermolecular interactions involved chains B and C, while chains $\mathrm{D}$ and $\mathrm{E}$ were removed from the crystal structure prior to docking. Polar hydrogens as well as Kollman charges were added on the amino acids. The charged protein was solvated using addsol utility in AutoDockTools.

\section{Docking simulation}

In Abbas et al. [22], the compounds in the methanolic leaf extract of $O$. spinosa were identified using liquid chromatography-mass spectrometry (LC-MS) analysis. These compounds were used to determine the docking simulation in this study. The grid parameter file was prepared using AutoDockTools to limit the search area, and a set of grid maps was created, using AutoGrid 4 (The Scripps Research Institute). A grid box was then utilized to select the area of protein structure to be mapped. The box size was set to $22.5,22.5$, and $22.5 \AA$ (x, y, and z, respectively). Docking simulations were carried out using the Lamarckian genetic algorithm parameters and 100 runs per simulation. After the completion of docking simulation, the output information of the docked coordinates and free energy of binding in the docking log file (dlg) were obtained from AutoDock. The 2D intermolecular interactions between TRPV1 (PDB ID: 5IS0) and campesterol, stigmasterol ononin and capsazepine were prepared by PoseView [23,24].

\section{Statistical analysis}

The normality test was conducted for all groups using Shapiro-Wilk test. The statistical significance of differences between groups was assessed by one-way analysis of variance, followed by Tukey's post-hoc test using GraphPad Prism version 7 (GraphPad Software, San Diego, CA). $P<$ 0.05 was considered significant. Data were presented as means \pm standard error of the mean.

\section{RESULTS}

\section{Effect of different treatments on CAPS-induced mechanical allodynia}

The injection of CAPS into the left hind paw of rats induced mechanical allodynia at all-time points (measured 

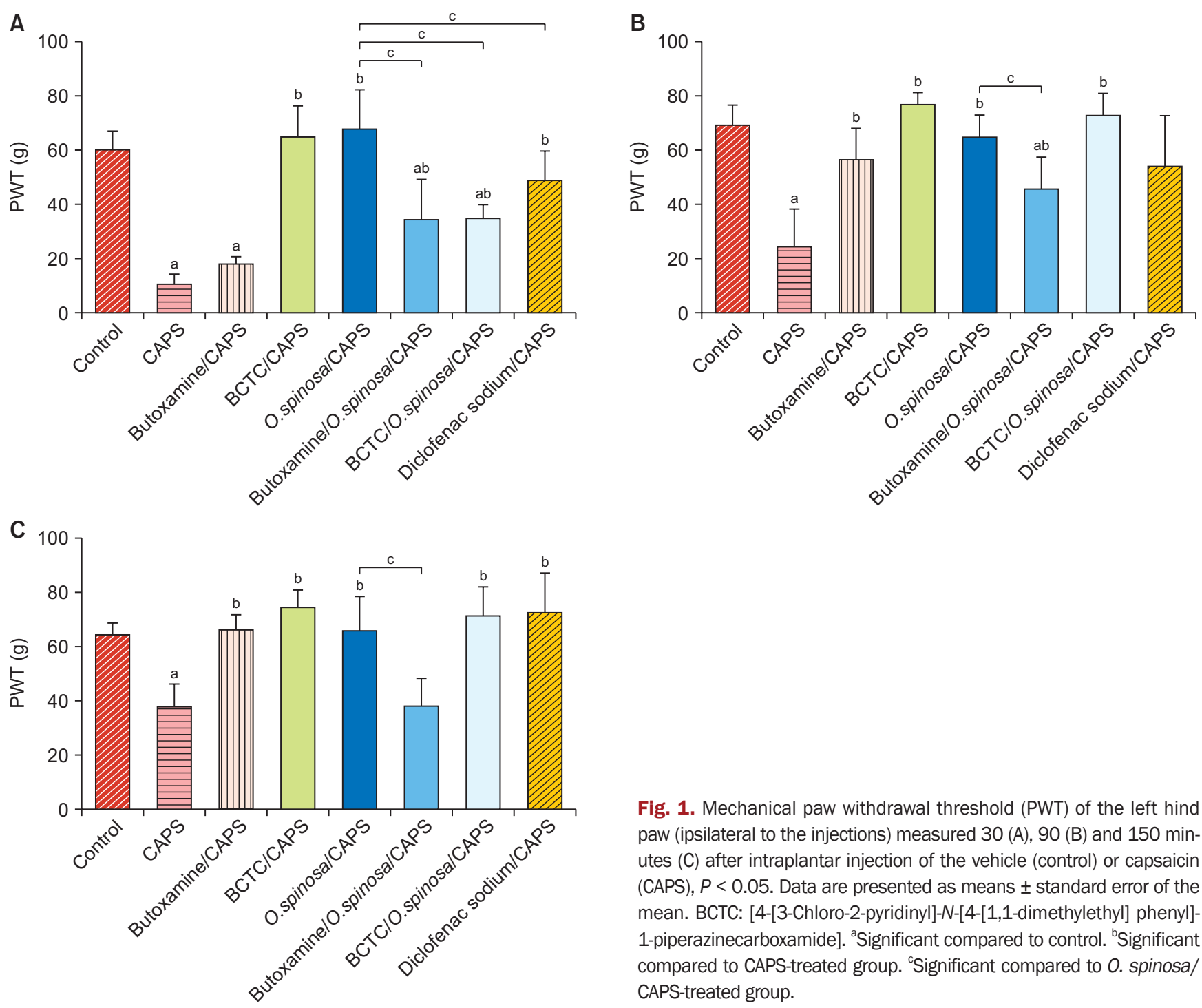

Fig. 1. Mechanical paw withdrawal threshold (PWT) of the left hind paw (ipsilateral to the injections) measured 30 (A), 90 (B) and 150 minutes $(C)$ after intraplantar injection of the vehicle (control) or capsaicin (CAPS), $P<0.05$. Data are presented as means \pm standard error of the mean. BCTC: [4-[3-Chloro-2-pyridinyl]-N-[4-[1,1-dimethylethyl] phenyl]1-piperazinecarboxamide]. ${ }^{a}$ Significant compared to control. ${ }^{b}$ Significant compared to CAPS-treated group. 'Significant compared to O. spinosa/ CAPS-treated group.

30,90 , or 150 min after CAPS injection) and decreased the PWT significantly compared to control group that received the vehicle (Fig. 1). Pre-injecting $100 \mu \mathrm{g} O$. spinosa methanolic extract into the same hind paw was effective in alleviating CAPS-induced mechanical allodynia at all-time points (Fig. 1). O. spinosa increased the PWT by 5.35 -fold compared to the 3.59-fold increase caused by diclofenac sodium. In addition, the anti-allodynic effect of $O$. spinosa in CAPS-induced mechanical allodynia decreased when the animals received $150 \mu \mathrm{g}$ butoxamine or $300 \mu \mathrm{g}$ BCTC prior to the ipl injection of the plant extract. This effect was exhibited at all time-points, 30, 90, and 150 minutes after CAPS injection (Fig. 1). Besides, the animals that received CAPS injection displayed guarding behavior for their paw compared to other groups. When PWT was measured in the right hind paw (contralateral to CAPS injection) in all groups, no effect was found at any time point (Fig. 2).

\section{Molecular docking}

All the proposed compounds in this study were successfully docked against TRPV1 (Table 1). The compounds that had the lowest binding energies are listed in Table 2 and Fig. 3. These compounds include campesterol, stigmasterol, and ononin with $-11.73,-11.02$, and $-8.91 \mathrm{kcal} / \mathrm{mol}$ binding energies, respectively. The results showed a conserved intermolecular interaction in these compounds, as well as the TRPV1 antagonist, capsazepine. The amino acids Leu515 and Leu553 were found to participate in the hydrophobic interaction with campesterol, stigmasterol, ononin, and capsazepine. Importantly Arg557 was shown to participate in the hydrogen bond interactions with previously mentioned compounds, except with capsazepine. Interestingly, the free energy of binding for the docked compounds is greatly affected by the number of residues that participate in hydrophobic interactions. 

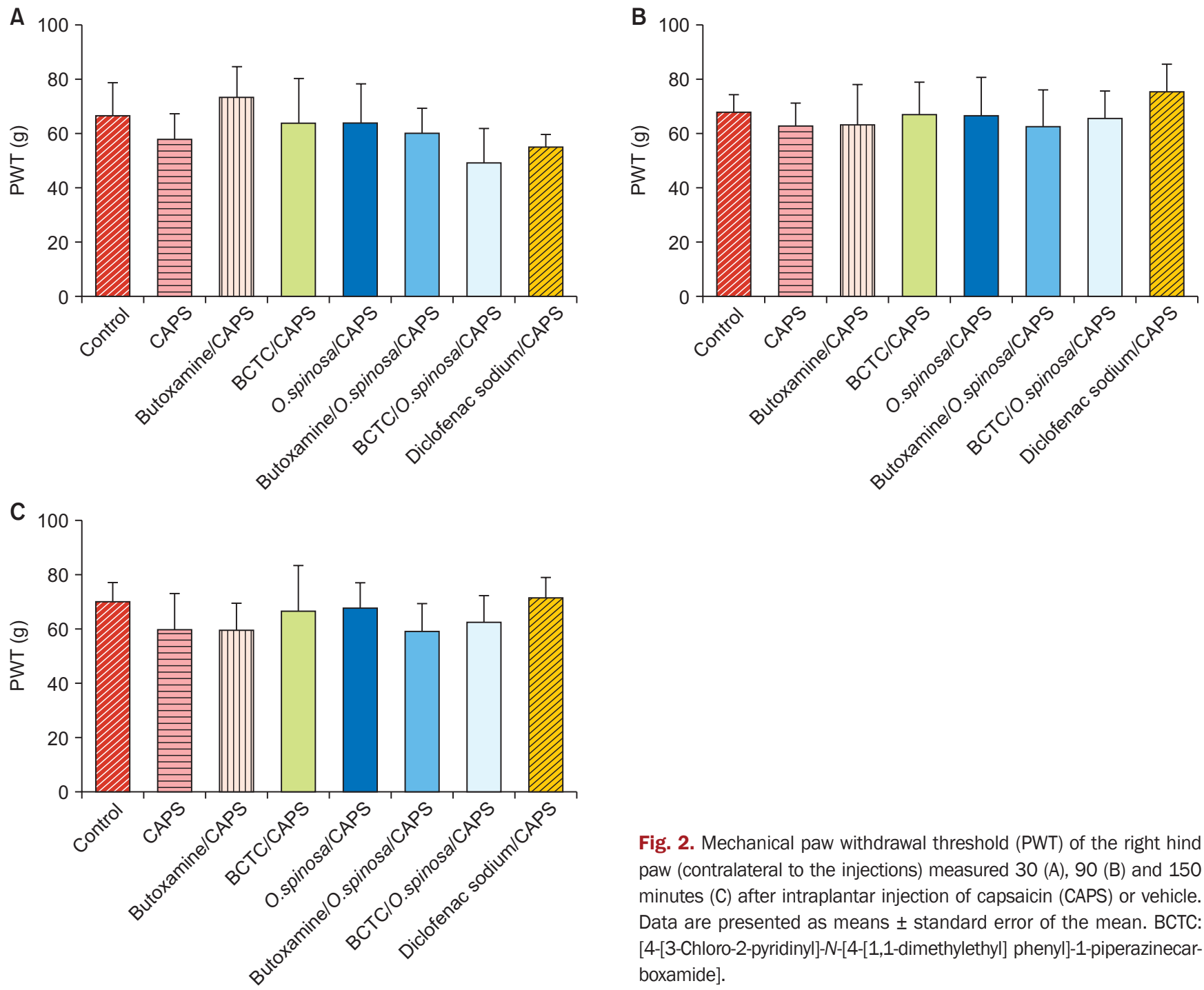

Fig. 2. Mechanical paw withdrawal threshold (PWT) of the right hind paw (contralateral to the injections) measured 30 (A), 90 (B) and 150 minutes $(C)$ after intraplantar injection of capsaicin (CAPS) or vehicle. Data are presented as means \pm standard error of the mean. BCTC: [4-[3-Chloro-2-pyridinyl]-N-[4-[1,1-dimethylethyl] phenyl]-1-piperazinecarboxamide].

\section{DISCUSSION}

It is well established that the activation of TRPV1 channel is responsible for mechanical allodynia [2]. In this study, the anti-allodynic activity of $O$. spinosa leaf extract was assessed in CAPS-induced mechanical allodynia in male Wistar rats. To the best of our knowledge, this is the first research reporting the effect of $O$. spinosa in alleviating CAPS-induced mechanical allodynia.

Ipsilateral injection of $O$. spinosa methanolic extract into the left hind paw, 20 minutes prior to CAPS injection, increased PWT in the animals by 5.35 -fold. Of note, the anti-allodynic activity of $O$. spinosa was stronger than the effect of the NSAID diclofenac sodium that was used as a positive control. In this study, the anti-allodynic action of O. spinosa in CAPS-induced mechanical allodynia model was attenuated using BCTC, a TRPV1 antagonist, indicating that its mechanism of action involves TRPV1 modulation. Furthermore, the decrease in PWT observed in the

group that received BCTC/O. spinosa/CAPS compared to the BCTC/CAPS-treated group suggests that the plant extract can be an antagonist that binds allosterically to TRPV1 channel or an inverse agonist for TRPV1. Another possibility is that the effect of the plant extract is implicated in influencing the availability of the channel on the plasma membrane, and this effect can be direct or indirect. Further research is needed to clarify these points. Additionally, the ipsilateral pretreatment with the $\beta 2$ adrenoreceptor antagonist, butoxamine, decreased the effect of $O$. spinosa on CAPS-induced mechanical allodynia, suggesting that the mechanism involves $\beta 2$ adrenoreceptor. No effect was found on the contralateral hind paw meaning that the anti-allodynic effect of $O$. spinosa extract was local and not systemic.

Interestingly, previous studies have investigated the interaction between $\beta 2$ adrenoreceptors and TRPV1 $[25,26]$. It is well established that $\beta$-adrenoreceptors are coupled to $\mathrm{G}$ stimulatory protein that enhances adenylate cyclase ac- 
tivity and intracellular cyclic adenosine monophosphate (cAMP), leading to the activation of protein kinase A, PKA (a cAMP-dependent protein kinase) [27]. Therefore, it is expected that $\beta 2$ adrenoreceptor blockers will deactivate PKA, thereby reducing TRPV1 activity and its availability in the plasma membrane, finally elevating PWT in the CAPS-induced mechanical allodynia model. This may explain the findings in the present study in which a $\beta 2$ blocker (butoxamine) decreased the anti-allodynic action of $O$. spinosa in CAPS-induced mechanical allodynia.

Notably, there are several receptors in the noradrenergic system whereby the effect of the noradrenergic system depends on the subtype of the receptors involved. Noradrenaline reduced the amplitudes of the inward CAPS currents

Table 1. Free energy of binding among compounds found in Ononis spinosa leaf extract and the co-crystallised ligand (capsazepine)

\begin{tabular}{lcc}
\hline \multicolumn{1}{c}{ Compound } & $\%$ & $\begin{array}{c}\text { Free energy of } \\
\text { binding } \\
\text { (kcal/mol) }\end{array}$ \\
\hline Campesterol & & -11.73 \\
Stigmasterol & 1.9 & -11.02 \\
Cycloartenol & 2.7 & -9.59 \\
Ononin & 5.1 & -8.91 \\
Medicarpin-3-o-glucoside & 20.5 & -8.84 \\
Biochanin-A & 1.8 & -8.46 \\
Biochanin & 0.9 & -8.44 \\
(-)-Maackiain-3-o-glucoside/Trifolirhizin & 1.0 & -8.34 \\
Rhoifolin & 6.4 & -8.28 \\
Formononetin & 3.6 & -8.03 \\
Tectorigenin & 3.8 & -7.91 \\
Apigenin & 3.3 & -7.52 \\
Quercetin & 1.5 & -7.07 \\
Quercitrin & 5.1 & -7.07 \\
Ferulic-acid & 3.0 & -7.03 \\
Genistein & 2.6 & -6.90 \\
Kaempferol & 1.8 & -6.69 \\
Myricitrin & 0.88 & -6.31 \\
Vitexin & 6.1 & -6.31 \\
Gentisic-acid & 0.66 & -6.16 \\
Gallic-acid & 5.4 & -5.90 \\
Rutoside & 4.6 & -5.59 \\
Hyperoside & 0.95 & -5.57 \\
\hline
\end{tabular}

in DRG neurons by either yohimbine (an antagonist for $\alpha 2$ adrenergic receptor) or propranolol (a $\beta$ adrenoreceptor antagonist) [25]. Previous reports showed the involvement of PKA and protein kinase C (PKC) in the inhibitory effect of $\alpha 2$ adrenergic receptors on TRPV1 [25]. However, it was found that $\alpha 2$ adrenergic receptors inhibited the function of TRPV1 in DRG neurons in a mechanism that involves calmodulin-dependent kinase II [28].

Earlier reports showed the involvement of $\beta 2$ adrenoreceptors in pain modulation, generally. In more detail, inhibition of catechol-O-methyltransferase, the enzyme that degrades catecholamines, increased pain sensitivity through activation of both $\beta 2$ - and $\beta 3$-adrenergic receptors [29]. Also, epinephrine caused mechanical hyperalgesia mediated by $\beta$ adrenoreceptor and an in vitro sensitization of rat nociceptors [15]. Furthermore, the interaction between $\beta 2$ adrenoreceptors and TRPV1 was reported in DRG neurons in a model of visceral hypersensitivity [30]. Catecholamines produced a significant reduction in TRPV1 desensitization in cultured DRG neurons, and this effect was mediated by $\alpha 1-, \alpha 2-$, and $\beta 2$-adrenoreceptors [26]. According to Coutaux et al. [31], epinephrine and norepinephrine do not normally activate nociceptors, but sensitize them under specific conditions, generating hyperalgesia. This sensitizing effect is probably mediated by protein kinases, mainly PKA and, to a lesser extent, PKC $[15,31]$. Therefore, the ability of butoxamine, in the present study, to abolish the anti-nociceptive effect of $O$. spinosa is most probably through kinases rather than a direct effect on TRPVl channel.

Importantly, the anti-nociceptive effect of $O$. spinosa was investigated previously in thermal models including tail flick and hot plate tests, as well as chemical pain models such as formalin and writhing tests $[10,13]$. However, it should be kept in mind that there are differences between the mechanisms of mechanical allodynia and thermal hyperalgesia at different levels [32]. In more detail, the neurons that transmit the signals from mechanoreceptors synapse at the superficial lamina of the spinal cord, while the signals from thermoreceptors synapse at the superficial and deep laminae of the spinal cord [32].

The results of the behavioral assay used in this study

Table 2. Top compounds in Ononis spinosa leaf extract having the lowest free energy of binding and with co-crystallised ligand (capsazepine)

\begin{tabular}{|c|c|c|c|}
\hline Compound & $\begin{array}{c}\text { Free energy of } \\
\text { binding } \\
(\mathrm{kcal} / \mathrm{mol})\end{array}$ & $\begin{array}{l}\text { Residues involved in } \\
\text { hydrogen bond interactions }\end{array}$ & Residues involved in hydrophobic interactions \\
\hline Campesterol & -11.73 & Arg557 & Ser512, Leu515, Thr550, Ala546, Leu553, Met547, Phe587, Phe591, and Leu669 \\
\hline Stigmasterol & -11.02 & Arg557 and Gln700 & Thr550, Leu515, Leu553, Ala566, and Glu570 \\
\hline Ononin & -8.91 & Tyr511 and Arg557 & Leu515, Leu553, Ala566, and lle573 \\
\hline Capsazepine & $(\mathrm{Ki}=120 \mathrm{nM})$ & Glu570 & Leu515, Met547, and Leu553 \\
\hline
\end{tabular}


A

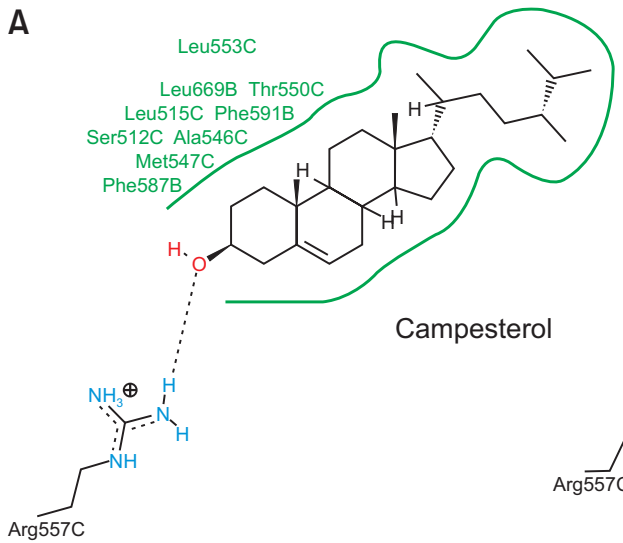

C

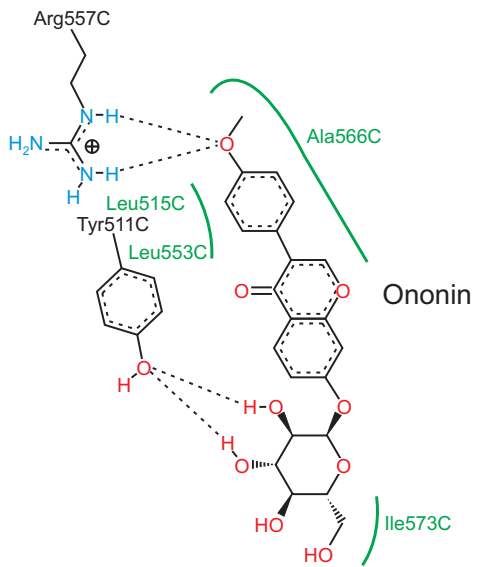

B

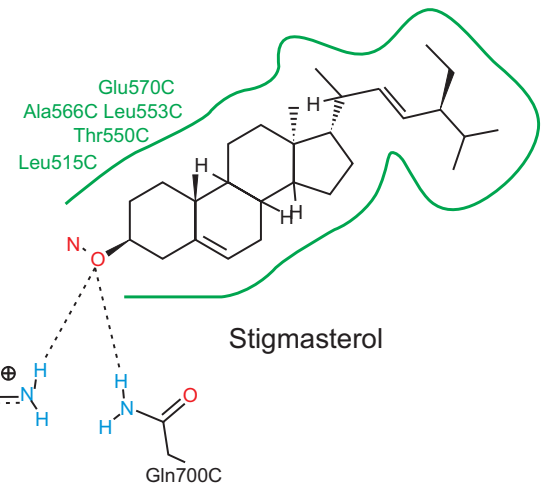

Fig. 3. Representations of the two dimensional intermolecular interactions between transient receptor potential vanilloid 1 (PDB ID: 5ISO) and (A) campesterol, (B) stigmasterol, (C) ononin, and (D) capsazepine. showed the efficacy of $O$. spinosa in blocking TRPV1, and the substance or group of substances responsible for that effect were specified. The molecular docking tool (AutoDock) selects the compounds that have the potential to bind proteins and is therefore a forward-looking plan towards improving and developing active small molecules [33]. Molecular modelling aids in the drug discovery process and reduces experimental cost and time [33].

$O$. spinosa belongs to the family Fabaceae that is rich in isoflavonoids (phytoestrogens) [34]. In fact, the crude extract includes different constituents that may function on different receptors and exert synergistic, additive, or antagonistic effects. In a previous study conducted in our laboratory, the ingredients of the methanolic leaf extract of $O$. spinosa were determined using LC-MS analysis [22]. Among these ingredients, the present study shows that several compounds were found to interact with TRPV1 based on molecular docking. Campesterol, stigmasterol, and ononin exhibited the strongest effect in this interaction, whereby ononin alone comprises almost a fifth of the compounds $(20.5 \%)$ in $O$. spinosa extract [22]. According to Peres et al. [35], the phytosterols campesterol and stigmasterol had analgesic effects in a writhing test. Of note, acetic acid injection in the writhing test causes abdominal constriction and trunk twisting [36]. This test involves the activation of acid-sensitive ion channels and TRPV1 channel in sensory primary fibers [37]. In another study, it was found that stigmasterol markedly attenuated the mechanical allodynia caused by surgical incision, partial sciatic nerve ligation, and complete Freund's adjuvant [38]. All these studies justify the effect of $O$. spinosa on CAPSinduced mechanical allodynia.

The present study sheds the light on the importance of $O$. spinosa extract in modulating TRPV1 action in mechanical allodynia. However, it raises many questions that need further investigation: 1 . What are the extract's constituents that block $\beta 2$ adrenoreceptor? 2. What are the effects of campesterol, stigmasterol, and ononin on TRPV1 in electrophysiological studies? 3 . What are the functions of these compounds in binding studies with TRPV1, are they antagonists or inverse agonists? Future research may answer these questions.

In conclusion, $O$. spinosa alleviated CAPS-induced mechanical allodynia through 2 mechanisms: the direct modulation of TRPV1 and the involvement of $\beta 2$ adrenoreceptor signaling. Further clinical studies are needed to confirm the effectiveness and safety of $O$. spinosa as an analgesic in human. 


\section{ACKNOWLEDGMENTS}

This work was published with the support of Al-Ahliyya Amman University, Amman, Jordan. The authors acknowledge the University of Jordan.

\section{CONFLICT OF INTEREST}

No potential conflict of interest relevant to this article was reported.

\section{FUNDING}

No funding to declare.

\section{ORCID}

Sahar Majdi Jaffal, https://orcid.org/0000-0001-7115-5841

Belal Omar Al-Najjar, https://orcid.org/0000-0001-6811-1792

Manal Ahmad Abbas, https://orcid.org/0000-0002-8962-1879

\section{REFERENCES}

1. Moran MM, Szallasi A. Targeting nociceptive transient receptor potential channels to treat chronic pain: current state of the field. Br J Pharmacol 2018; 175: 2185-203.

2. Cavanaugh DJ, Chesler AT, Bráz JM, Shah NM, Julius D, Basbaum AI. Restriction of transient receptor potential vanilloid-1 to the peptidergic subset of primary afferent neurons follows its developmental downregulation in nonpeptidergic neurons. J Neurosci 2011; 31: 10119-27.

3. Gilchrist HD, Allard BL, Simone DA. Enhanced withdrawal responses to heat and mechanical stimuli following intraplantar injection of capsaicin in rats. Pain 1996; 67: 179-88.

4. Caterina MJ, Schumacher MA, Tominaga M, Rosen TA, Levine JD, Julius D. The capsaicin receptor: a heat-activated ion channel in the pain pathway. Nature 1997; 389: 816-24.

5. Julius D. TRP channels and pain. Annu Rev Cell Dev Biol 2013; 29: 355-84.

6. Alsalem M, Wong A, Millns P, Arya PH, Chan MS, Bennett A, et al. The contribution of the endogenous TRPV1 ligands 9-HODE and 13-HODE to nociceptive processing and their role in peripheral inflammatory pain mechanisms. Br J Pharmacol 2013; 168: 1961-74.

7. Wongrakpanich S, Wongrakpanich A, Melhado K, Rangaswami J. A comprehensive review of non-steroidal antiinflammatory drug use in the elderly. Aging Dis 2018; 9: 14350 .
8. Al-Eisawi DMH. Field guide to wild flowers of Jordan and neighbouring countries. Amman, Jordan Press Foundation. 1998.

9. Al-Snafi AE. The traditional uses, constituents and pharmacological effects of Ononis spinosa. IOSR J Pharm 2020; 10: 53-9.

10. Yõlmaz BS, Özbek H, Çitoğlu GS, Uğraş S, Bayram İ, Erdoğan E. Analgesic and hepatotoxic effects of Ononis spinosa L. Phytother Res 2006; 20: 500-3.

11. Ergene Öz B, Saltan İşcan G, Küpeli Akkol E, Süntar İ, Keleş H, Bahadır Acıkara Ö. Wound healing and anti-inflammatory activity of some Ononis taxons. Biomed Pharmacother 2017; 91: 1096-105.

12. Al-Khalil S. A survey of plants used in Jordanian traditional medicine. Int J Pharmacogn 1995; 33: 317-23.

13. Jaffal SM, Abbas MA. Antinociceptive action of Ononis spinosa leaf extract in mouse pain models. Acta Pol Pharm 2019; 76: 299-304.

14. Lolignier S, Eijkelkamp N, Wood JN. Mechanical allodynia. Pflugers Arch 2015; 467: 133-9.

15. Khasar SG, McCarter G, Levine JD. Epinephrine produces a beta-adrenergic receptor-mediated mechanical hyperalgesia and in vitro sensitization of rat nociceptors. J Neurophysiol 1999; 81: 1104-12.

16. Nozadze I, Tsiklauri N, Gurtskaia G, Tsagareli MG. NSAIDs attenuate hyperalgesia induced by TRP channel activation. Data Brief 2016; 6: 668-73.

17. Ferrier J, Marchand F, Balayssac D. Assessment of mechanical allodynia in rats using the electronic von frey test. Bio Protoc 2016; 6: e1933.

18. Gao Y, Cao E, Julius D, Cheng Y. TRPVl structures in nanodiscs reveal mechanisms of ligand and lipid action. Nature 2016; 534: 347-51.

19. Walpole CS, Bevan S, Bovermann G, Boelsterli JJ, Breckenridge $\mathrm{R}$, Davies JW, et al. The discovery of capsazepine, the first competitive antagonist of the sensory neuron excitants capsaicin and resiniferatoxin. J Med Chem 1994; 37: 1942-54.

20. ACD/Labs. ACD/Structure Elucidator [computer program]. Version 2018.1 Toronto (ON).

21. BIOVIA, Dassault Systèmes. BIOVIA discovery studio visualizer [computer program]. Version 2002-2021 San Diego (CA).

22. Abbas MA, Kandil YI, Abbas MM. Efficacy of extract from Ononis spinosa L. on ethanol-induced gastric ulcer in rats. J Tradit Chin Med 2021; 41: 270-5.

23. Fricker PC, Gastreich M, Rarey M. Automated drawing of structural molecular formulas under constraints. J Chem Inf Comput Sci 2004; 44: 1065-78.

24. Stierand K, Maass PC, Rarey M. Molecular complexes at a glance: automated generation of two-dimensional complex diagrams. Bioinformatics 2006; 22: 1710-6.

25. Matsushita Y, Manabe M, Kitamura N, Shibuya I. Adrenergic receptors inhibit TRPV1 activity in the dorsal root ganglion 
neurons of rats. PLoS One 2018; 13: e0191032.

26. Filippi A, Caruntu C, Gheorghe RO, Deftu A, Amuzescu B, Ristoiu V. Catecholamines reduce transient receptor potential vanilloid type 1 desensitization in cultured dorsal root ganglia neurons. J Physiol Pharmacol 2016; 67: 843-50.

27. Jeske NA, Diogenes A, Ruparel NB, Fehrenbacher JC, Henry $\mathrm{M}$, Akopian AN, et al. A-kinase anchoring protein mediates TRPV1 thermal hyperalgesia through PKA phosphorylation of TRPV1. Pain 2008; 138: 604-16.

28. Chakraborty S, Elvezio V, Kaczocha M, Rebecchi M, Puopolo M. Presynaptic inhibition of transient receptor potential vanilloid type 1 (TRPV1) receptors by noradrenaline in nociceptive neurons. J Physiol 2017; 595: 2639-60.

29. Nackley AG, Tan KS, Fecho K, Flood P, Diatchenko L, Maixner W. Catechol-O-methyltransferase inhibition increases pain sensitivity through activation of both beta2and beta3-adrenergic receptors. Pain 2007; 128: 199-208.

30. Zhu L, Zhao L, Qu R, Zhu HY, Wang Y, Jiang X, et al. Adrenergic stimulation sensitizes TRPV1 through upregulation of cystathionine $\beta$-synthetase in a rat model of visceral hypersensitivity. Sci Rep 2015; 5: 16109.

31. Coutaux A, Adam F, Willer JC, Le Bars D. Hyperalgesia and allodynia: peripheral mechanisms. Joint Bone Spine 2005;
72: $359-71$.

32. Benarroch EE. Dorsal horn circuitry: complexity and implications for mechanisms of neuropathic pain. Neurology 2016; 86: 1060-9.

33. Al-Najjar BO. Synthesis, molecular docking and antioxidant evaluation of benzylidene ketone derivatives. Jordan J Biol Sci 2018; 11: 307-13.

34. Benedec D, Vlase L, Oniga I, Toiu A, Tămaş M, Tiperciuc B. Isoflavonoids from Glycyrrhiza sp. and Ononis spinosa. Farmacia 2012; 60: 615-20.

35. Peres MTLP, Monache FD, Pizzolatti MG, Santos ARS, Beirith A, Calixto JB, et al. Analgesic compounds of Croton urucurana Baillon. Pharmaco-chemical criteria used in their isolation. Phytother Res 1998; 12: 209-11.

36. Lee CW. Introduction of visceral pain model to test of visceral nociception in the rats. J Korean Pain Soc 1995; 8: 25-30.

37. Julius D, Basbaum AI. Molecular mechanisms of nociception. Nature 2001; 413: 203-10.

38. Walker CIB, Oliveira SM, Tonello R, Rossato MF, da Silva Brum E, Ferreira J, et al. Anti-nociceptive effect of stigmasterol in mouse models of acute and chronic pain. Naunyn Schmiedebergs Arch Pharmacol 2017; 390: 1163-72. 